\title{
The relationships between rugby ground pass accuracy and kinematic variables resulting from two different pelvic orientations
}

\author{
A Green, ${ }^{1} \mathrm{PhD}$, $\mathbf{S}$ Kerr, ${ }^{1} \mathrm{PhD}$, B Olivier, ${ }^{2} \mathrm{PhD}, \mathrm{C}$ Dafkin, ${ }^{1} \mathrm{MSc}$, \\ W McKinon, 1 PhD \\ 1 School of Physiology, Faculty of Health Sciences, University of the \\ Witwatersrand, Johannesburg, South Africa
}

2 Department of Physiotherapy, School of Therapeutic Sciences, Faculty of Health Sciences, University of the Witwatersrand, Johannesburg, South Africa

\section{Corresponding author: A Green (Andrew.Green@students.wits.ac.za)}

Introduction: Despite having been largely understudied, one of the crucial components of a team's success in rugby is accurate passing. This study identified biomechanical correlates of the rugby ground pass and accuracy performance.

Methods: Sixteen club players (height $1.77 \pm 0.04 \mathrm{~m}$; mass $86.8 \pm 16.8 \mathrm{~kg}$ ) undertook a combined total of 96 passes and their respective body kinematics were analysed concurrent with measurements of pass accuracy at $10 \mathrm{~m}$. Two distinct types of body orientations were found to be utilised by the players: a side-on orientation (pelvic rotation $>80^{\circ}$ ) and a front-on orientation (pelvic rotation $<80^{\circ}$ ).

Results: Side-on body orientation passes were more accurate than front-on body orientation passes $(\mathrm{p}<0.0001)$. Fair relationships were present between the pass accuracy and upper body and hip kinematics for the two distinct body orientations individually. However, no common relationships were observed between the different orientations.

Conclusion: Therefore different strategies exist within players to perform the ground pass with varying grades of accuracy.

Keywords: biomechanics, direct measurement

S Afr J Sports Med 2016;28(2):51-54. DOI: 10.17159/2078-516X/2016/v28i2a421

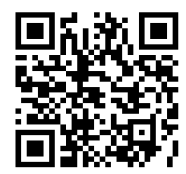

All rugby players are required to be proficient at passing the ball (unlike specialist areas distinct to rugby such as the scrum and lineout), variation in passing ability among playing positions has been reported [1]. Current research has highlighted the effect of hand dominance on the rugby pass, both maximally [2] and over various set distances [3]. Recent studies have shown that training is known to influence passing dynamics ${ }^{[4]}$ and that caffeine supplementation does not influence passing accuracy ${ }^{[5]}$. Interestingly, these studies have looked exclusively at the running pass. Another passing type in rugby is the pass from the ground. When a scrum or breakdown occurs the ball is placed on the ground where the arriving player will attempt to distribute the ball to another advancing player. The ground pass is made from a comparatively static position, when compared to the running pass. Regarding the technical attributes of the ground pass there are two different strategies that are commonly employed: 1) a technique that predominantly uses the arms; or 2) a technique that predominantly uses rotations of the shoulder and pelvic girdles. The optimal passing sequence should involve both rotation of the torso and pelvis and the extension of the arms, resulting in an accurate pass.

The purpose of this study was therefore to primarily assess whether biomechanical correlates of the rugby ground pass accuracy exist in high-level amateur rugby players. Additionally, the study aims to assess the effects of the two passing strategies and their resulting accuracies. It was hypothesised that passing accuracy may be related to the upper body kinematics of the players, specifically the rotations of the torso and pelvic girdles along with additional contributions from the arms.

\section{Methods}

\section{Participants}

Sixteen first team university level rugby players (11 backs, 5 forwards) that volunteered for this study had an average age of $22 \pm 2$ years; height of $1.77 \pm 0.04 \mathrm{~m}$ and body mass of $86.8 \pm 16.8 \mathrm{~kg}$. Ethical approval was granted by the University Ethics Committee (M131019) and written informed consent was received prior to the start of testing. All participants were right-handed and injury-free at the time of the study.

The participants were required to pass from a distance of 10 $\mathrm{m}$ towards a target within a larger calibrated frame. The calibration frame consisted of a $2 \mathrm{~m} \times 2 \mathrm{~m}$ metal frame with a middle portion consisting of a rectangular target defined by the vertical limits in-between $0.74 \mathrm{~m}$ and $1.77 \mathrm{~m}$ from the ground. The horizontal width of the target was the length of a regulation rugby union ball $(0.33 \mathrm{~m})$. The vertical limits were based on data collected from 27 players, when asked what constituted the limits of a catchable pass while running on attack during a game. Pass accuracy was quantified as the distance of the ball position from the central point of the accurate zone. Digital video images were recorded (Sony DCR-SX41, Sony Corporation, Tokyo, Japan) and the position of the ball as it reached the frame was digitally identified using image analysis tools (MatLab 7, Mathworks, Natick, USA).

Full-body kinematics were recorded using an 18 camera system recording at $100 \mathrm{~Hz}$ (Optitrack flex:V100r2, Natural Point Inc., Corvallis, Oregon, USA). A measurement volume of approximately $18 \mathrm{~m}^{3}$ was calibrated (AMASS, C-Motion Germantown, Maryland, USA) in the area where ball release would occur, to a level of sub-millimetre error. Custom written algorithms were used to analyse body positions as derived from raw marker location data in MatLab 7. The various kinematic variables were measured based on descriptions outlined Table 1.

Passing kinematics were analysed at the moment of ball release. Two distinct passing groups were identified (Fig. 1): one group with a pelvic rotation angle of greater than $80^{\circ}$ between the direction of the target and the pelvic vector 
Table 1. Definitions of the kinematic variables

Kinematic Variable
Neck flexion

Fig. 1. Defining the two body orientations, by the pelvic positions at ball release. A. front-on orientation (pelvic rotation angle less than $80^{\circ}$ relative to the direction of the target). $B$. side-on orientation (pelvic rotation angle greater than $80^{\circ}$ relative to the direction of the target).

(side-on orientation) and one with a pelvic rotation angle of less than $80^{\circ}$ between the direction of the target and the pelvic vector (front-on orientation). The corresponding side of the participant's body to that of the intended pass direction will be considered as the pass side, and the opposite side will be considered the stance side.

\section{Procedure}

Participants underwent a self-guided warm-up prior to testing. All participants were allowed no more than five practise passes under the experimental conditions. All participants performed in a randomised order a total of six passes (three to the left, and three to the right) using a set of standardised training rugby union balls (Gilbert XT300, Grays of Cambridge (Int) Ltd, East Sussex, United Kingdom). Participants were instructed to pass legally (backwards or lateral) towards the target with the aim of achieving an accurate pass.

\section{Statistical analysis}

All data distributions were analysed using a Shapiro Wilk normality test. Passing accuracy error distance is represented as median: range (between quartile 1 and quartile 3) due to the non-normal distribution. Accordingly, a Mann-Whitney test was used to compare accuracy differences between the two passing orientation types. All kinematic data are represented as mean \pm standard deviation. Spearman's correlations were performed between the pass accuracy error distance and the kinematic variables at ball release in MatLab 7. A significance level of $p<0.05$ was applied. Positive values indicate that a greater kinematic variable would result in less accurate passes (larger error). Negative correlations indicate that a greater kinematic variable would result in more accurate passes (smaller error).

\section{Results}

The passing accuracy error was not significantly $(\mathrm{p}=0.945)$ different between the right direction $(20.0 \mathrm{~cm}$ : 8.4-44.9 cm) and the left direction $(20.9 \mathrm{~cm}: 9.1-43.8 \mathrm{~cm})$. The pass accuracy error distances were significantly larger $(\mathrm{p}<0.0001)$ for the front-on body orientation $(34.1 \mathrm{~cm}$ : 12.9-49.1 cm) $(\mathrm{n}=64)$ compared to the side orientation $8.8 \mathrm{~cm}$ : $4.4-20.3 \mathrm{~cm})(\mathrm{n}=32)$. Playing positions and individual passing accuracies are reported in Table 2. Additionally, Table 2 shows that only four participants used a single body orientation ( 3 front-on; 1 side-on) for their six passes. Leaving the remaining 12 participants with a combination of the two body orientations used during the pass. Correlations between body kinematics and the pass accuracy distances, and their qualitative descriptions, are reported in Table 3. 
Table 2. Playing positions and individual assessment of passing orientation frequency, median and inter-quartile range

\begin{tabular}{|c|c|c|c|c|c|}
\hline Participant & Playing position & $\begin{array}{l}\text { Front-on/ } \\
\text { Side-on }\end{array}$ & $\begin{array}{c}\text { Accuracy median } \\
(\mathrm{cm})\end{array}$ & $\begin{array}{c}\text { Accuracy quartile } 1 \\
(\mathrm{~cm})\end{array}$ & $\begin{array}{c}\text { Accuracy quartile } 3 \\
(\mathrm{~cm})\end{array}$ \\
\hline 1 & Scrum half & $3 / 3$ & 40.9 & 34.9 & 49.7 \\
\hline 2 & Scrum half & $6 / 0$ & 18.1 & 12.9 & 32.4 \\
\hline 3 & Flank & $5 / 1$ & 34.0 & 21.1 & 39.6 \\
\hline 4 & Wing & $3 / 3$ & 9.4 & 8.7 & 13.0 \\
\hline 5 & Prop & $6 / 0$ & 8.5 & 7.0 & 9.9 \\
\hline 6 & Hooker & $0 / 6$ & 26.9 & 24.3 & 28.9 \\
\hline 7 & Wing & $6 / 0$ & 4.9 & 3.9 & 5.2 \\
\hline 8 & Number 8 & $3 / 3$ & 5.8 & 5.3 & 8.4 \\
\hline 9 & Fly half & $3 / 3$ & 4.6 & 3.0 & 5.8 \\
\hline 10 & Fullback & $5 / 1$ & 43.1 & 30.6 & 45.4 \\
\hline 11 & Fly half & $5 / 1$ & 15.4 & 10.1 & 20.3 \\
\hline 12 & Prop & $5 / 1$ & 39.1 & 31.0 & 44.9 \\
\hline 13 & Wing & $3 / 3$ & 39.7 & 14.1 & 103.5 \\
\hline 14 & Centre & $3 / 3$ & 62.0 & 57.2 & 98.3 \\
\hline 15 & Centre & $5 / 1$ & 44.8 & 36.6 & 49.1 \\
\hline \multirow[t]{2}{*}{16} & Scrum half & $3 / 3$ & 29.0 & 18.3 & 45.2 \\
\hline & Combined & $64 / 32$ & 21.0 & 9.2 & 44.6 \\
\hline
\end{tabular}

Table 3. Kinematic joint angular data and Spearman's rank correlation coefficients for kinematic variables and their relationships to the pass accuracy error distance for front and side body orientations.

\begin{tabular}{|c|c|c|c|c|c|c|}
\hline & \multicolumn{2}{|c|}{ Front body orientation $(\mathrm{N}=64)$} & \multicolumn{4}{|c|}{ Side body orientation $(\mathrm{N}=32)$} \\
\hline & $\begin{array}{c}\text { Kinematic angle } \\
\left({ }^{\circ}\right)\end{array}$ & Spearman's $\mathbf{r}$ & $\begin{array}{c}\text { Qualitative } \\
\text { descriptions }+\end{array}$ & $\begin{array}{c}\text { Kinematic angle } \\
\left({ }^{\circ}\right)\end{array}$ & Spearman's r & $\begin{array}{c}\text { Qualitative } \\
\text { descriptions }+\end{array}$ \\
\hline Neck flexion & $100.2 \pm 13.4$ & 0.124 & little & $99.3 \pm 15.6$ & $-0.458^{*}$ & fair \\
\hline Head rotation & $88.4 \pm 9.5$ & $0.382^{*}$ & fair & $88.6 \pm 7.4$ & -0.154 & little \\
\hline Torso rotation & $32.4 \pm 18.6$ & -0.189 & little & $143.1 \pm 39.4$ & 0.122 & little \\
\hline Lateral bend & $15.9 \pm 8.1$ & 0.002 & little & $16.9 \pm 7.9$ & 0.269 & fair \\
\hline Back flexion & $15.7 \pm 8.2$ & 0.204 & little & $17.9 \pm 4.0$ & -0.027 & little \\
\hline Stance side elbow & $68.1 \pm 18.7$ & -0.258 & fair & $66.0 \pm 15.3$ & $0.655^{*}$ & moderate \\
\hline Pass side elbow & $50.2 \pm 17.6$ & $-0.331^{*}$ & fair & $52.9 \pm 12.6$ & 0.013 & little \\
\hline Stance side wrist & $149.1 \pm 15.6$ & -0.210 & little & $150.0 \pm 13.0$ & $-0.457^{*}$ & fair \\
\hline Pass side wrist & $127.2 \pm 22.7$ & 0.134 & little & $130.6 \pm 17.8$ & $-0.464^{*}$ & fair \\
\hline Pelvic rotation & $35.8 \pm 14.6$ & -0.105 & little & $133.8 \pm 33.2$ & -0.182 & little \\
\hline$X$-factor & $-2.7 \pm 12.7$ & $-0.380^{*}$ & fair & $6.7 \pm 14.8$ & 0.140 & little \\
\hline Stance side knee & $58.1 \pm 27.5$ & 0.050 & little & $57.0 \pm 19.9$ & -0.255 & fair \\
\hline Pass side knee & $53.6 \pm 28.3$ & 0.055 & little & $53.5 \pm 24.0$ & -0.095 & little \\
\hline Stance side ankle & $75.2 \pm 16.4$ & 0.096 & little & $81.4 \pm 19.1$ & 0.253 & fair \\
\hline Pass side ankle & $65.4 \pm 8.8$ & 0.111 & little & $64.5 \pm 6.0$ & -0.118 & little \\
\hline
\end{tabular}

+ Qualitative descriptions for the strength of the relationships were defined as Portney and Watkins ${ }^{[6]}$ : $\mathrm{r}=0.00-0.25$ little or no relationship; $\mathrm{r}=0.25-0.50$ fair relationship; $r=0.50-0.75$ moderate to good relationship; $r>0.75$ good to excellent relationship.

${ }^{*} \mathrm{p}<0.05$ Spearman's rank correlation coefficient

\section{Discussion}

Unlike previous studies that investigated the running pass in rugby players ${ }^{[1-5]}$, the current study aimed to assess kinematic strategies and accuracy performance of the rugby union ground pass. It was noted that two distinct types of body orientations were utilised by the players. These two distinct types of body orientations resulted in differences in accuracy performance and kinematic correlations to passing accuracy. 
Firstly, it must be noted that there was no significant difference between the passes directed to the right and the passes directed to the left. This is contrary to studies presented by Pavely et al. ${ }^{[2]}$ Worsfold and Page ${ }^{[3]}$ and Sayers and Ballon ${ }^{[7]}$. These studies reported the effects of hand dominance on maximal pass distance, various pass distances and pass velocity, respectively. The lack of difference in passing accuracy based on hand dominance resulted in the combined accuracies of the passes being used. From this analysis it was noted that two distinct body orientations were used to execute the passes: front-on and side-on. However, only four of the 16 participants used a single body orientation to execute all of their six passes. The remaining 12 participants all used a combination of front-on and sideon body orientations.

The significant correlations highlight interesting relationships between the body movements and pass accuracy independent of the two distinct body orientations. Isolating the front-on body orientation, the positive correlation for accuracy and head rotation would suggest the importance of identifying the target in executing an accurate pass. Furthermore, the front-on body orientation requires a greater extension of the pass side elbow, while the side-on body orientation requires the stance elbow flexion to be larger to achieve accurate passes. A significant correlation between the velocity of the stance side elbow flexion and the resulting pass velocity has been reported [7]. Consequently, the stance side elbow may be essential to the performance of the pass. Interestingly, the passes were more accurate with decreasing values of $\mathrm{X}$-factor. This would suggest a greater torso rotation relative to the pelvic rotation is needed to achieve an accurate pass. Regarding the side-on body orientation relationships: pass accuracy requires more neck extension and bilateral wrist flexion, as indicated by the significant correlations. These results may indicate that in this body orientation, the players would need to elevate their heads to observe and identify the target by reducing the degree of neck flexion (increased neck extension).

The relationships shown for the pass accuracy and the different body orientations highlight the potentially different strategies that are used to achieve an accurate pass. Interestingly, there were no common kinematic variables that were significantly correlated to accuracy between the two different body orientations. It would appear that in the side-on orientation the players would rely on the arms, specifically the stance elbow flexion, head flexion, stance side wrist flexion and pass side wrist flexion. While the front-on body orientation utilises head rotation, pass side elbow flexion and $\mathrm{X}$-factor (torso and pelvic girdle separation) to achieve accurate passes. While these relationships do give some insight into the different strategies used in the two distinct pass styles, they do not definitively identify all the parameters used by the players. Furthermore, they merely identify fair relationships between the parameters and do not imply causation. Further investigation, specifically into the muscular activity, is required to conclusively answer this. Ultimately, the passing type used by the players should not affect the gameplay provided they are able to achieve an accurate pass. The data within the current study would suggest that the side-on pass orientation was shown to be more accurate than the front-on pass. However, the accuracy constraints in the current study do not take into account any movement of the receiving player, with the vast majority of passes likely to be caught by the receiving player. Additional limitations include: the small sample size; playing level of the participants; and the limited number of passes performed by each player. Future studies are warranted to investigate the duration of the passes and the effects of playing position on the body orientations used to perform the passes.

\section{Conclusion}

The current study has identified two different kinematic passing strategies used by players to achieve different levels of pass accuracy. Specifically, the side-on body orientation produced more accurate passes than the front-on body orientation. Coaches should train ground passing strategies that result in the most accurate outcomes. The majority of ground passes performed by a sample of rugby players of various positions resulted in a front-on body orientation. However, when a side-on pass orientation was used, the passes were significantly more accurate. Passing strategies may be reliant on the orientation of the body relative to the target.

\section{Take home message}

Ground passing accuracies were not subject to the effect of hand dominance over $10 \mathrm{~m}$. Passing accuracies may be reliant on the body orientations used during the passing sequence and fair-to-moderate relationships exist between passing accuracy and kinematic variables. Training regimes that enhance these kinematic variables may be beneficial to improving passing accuracy.

\section{References}

1. Gabbett T, Kelly J, Pezet T. A comparison of fitness and skill among playing positions in sub-elite rugby league players. J Sci Med Sport 2008;11(6):585-592. [http://dx.doi.org/10.1016/j.jsams.2007.07.004]

2. Pavely S, Adams RD, Di Francesco TI, et al. Execution and outcome differences between passes to the left and right made by first-grade rugby union players. Phys Ther Sport 2009;10(4):136-141. [http://dx.doi.org/10.1016/j.ptsp.2009.05.006]

3. Worsfold PR, Page M. The influences of rugby spin pass technique on movement time, ball velocity and passing accuracy. Int J Perform Anal Sport 2014;14(1):296-306. http://www.ingentaconnect.com/content/uwic/ujpa/2014/00000014/0 0000001/art00026

4. Hooper JJ, James SD, Jones DC, et al.. The influence of training with heavy rugby balls on selected spin pass variables in youth rugby union players. J Sci Med Sports 2008;11(2): 209-213. [http://dx.doi.org/10.1016/j.jsams.2006.09.005]

5. Assi $\mathrm{HN}$, Bottoms L. The effects of caffeine on rugby passing accuracy while performing the Reactive Agility Test. Sci Sport 2014;29(5):275-281. [http://dx.doi.org/10.1016/j.scispo.2014.07.012]

6. Portney LG, Watkins MP. (2009). Foundations of clinical research: Applications to practice. 3rd ed.. London: Pearson Prentice Hall.

7. Sayers M, Ballon R. Biomechanical analysis of a rugby pass from the ground. 7th World Congress of Science and Football, Nagoya, Japan 26-30 May 2011. 